\title{
A Hybrid Modified Approach for Solving Fuzzy Differential Equations
}

\author{
Alauldeen N. Ahmed, Basma A. Nema* \\ College of Science Department, Al-Nahrain University \\ Baghdad-Iraq \\ ${ }^{*}$ Corresponding author's email: Bsmaa91b [AT] gmail.com
}

\begin{abstract}
In this paper, a hybrid method is presented by combining Laplace transformation and variational iteration method for solving fuzzy differential equations with fuzzy initial and boundaries values. A defuzzification technique had been implemented to convert the fuzzy parameters into crisp values by building an extended ranking method. Then the method is implemented on the new problem in which two approaches has been built according to the formula of Lagrange multiplier obtaining the lower, upper and center solutions.
\end{abstract}

Keywords-Fuzzy differential equation, Laplace transformation, Variational iteration methods, Lagrange multiplier

\section{INTRODUCTION}

Differential equations have been widely explored in many fields,from applications in physics, engineering, economics, and biology to theoretical mathematical developments A much newer theory, fuzzy sets theory, created to model subjective concepts whose boundaries are non-sharp, has also been explored in various fields due to its great applicability and functionality. As soon as the idea of a function with fuzzy values was born, it raised the idea of a fuzzy differential equation (FDE). Since then, researches given rise to different theories of FDEs. Zadeh [1] introduced the notion of inclusion, union, intersection, complement, relation, and convexity of the Fuzzy set. The original definition of fuzzy sets is to consider a class of objects with a continuum grade of membership function which assigns to each object a grade of membership value between zero and one. The differentiability and inerrability properties of fuzzy-set-value damping of a real variable were studied by Kaleva [2], he gave an existence and uniqueness theorem for a solution to a fuzzy differential equation. The twopoints boundary value problem for a second order fuzzy differential equation introduced by Khastan and Nieto [3]. They used a generalized differentiability concept. They presented a new concept of solutions and, utilizing the generalized differentiability. Ahmad et al [4] proposed a natural way to model dynamic systems under uncertainty. A fuzzy boundary value problem (FBVPs) was used and related uncertain systems. The fuzzy Laplace transform was used to find the solution of two-point boundary value under generalized Hukuhara differentiability. The Fuzzy Laplace Transformation for the nth derivative of a fuzzy valued function named as nth derivative theorem were generalized by Ahmad et al, [5]. The analytical solution method for the solution of an nth order fuzzy initial value problem was introduced. They showed that this method was a simple approach toward the solution of nth order fuzzy initial value problem by the nth generalized form. They solved any order of FIVP by their method.

In recent years approximate-analytical methods such as Homotopy Perturbation Method (HPM), Variational Iteration Method (VIM), and Adomian Decomposition Method (ADM) have been used to solve fuzzy initial value problems which inclose ordinary differential equations. Ghanbari [6] used the HPM to solve first order linear fuzzy initial value problems involving ordinary differential equations. The use of ADM for solving first order linear and nonlinear fuzzy initial value problems was introduced by the authors of $[7,8,9]$. While the variational iteration method (VIM) was developed by He [10, 11] for solving nonlinear problems, it has been successful applied on initial and boundary value problems. The fuzzy variational iteration method (FVIM) has been applied by Jafari [12] to finding solutions of nth order linear fuzzy differential equations. The solution obtained by the variational iteration method was an infinite power series, which, with appropriate initial condition, can be expressed in a closed form, i.e. the exact solution. The results presented in this contribution showed that the variational iteration method was a powerful mathematical tool to solving nth order fuzzy differential equations. For linear fuzzy differential equation, its exact solution can be obtained by the VIM due to the fact that the Lagrange multiplier can be exactly identified.

The objective of this work is to solve the nth order Fuzzy difference equations via a combination of Laplace transforms and variational iteration method. In which the solution nth order Fuzzy difference equations were obtained by using fuzzy initial conditions and fuzzy boundary conditions. 


\section{PRELIMINARIES}

In this section, the basic concepts of fuzzy set theory, and some necessary definitions are presented.

Definition 2.1 [13]:

The universal set $U$ is a classical set of objects and whose generic elements are denoted by $x$. The membership in a classical subset $A$ of $U$ is often viewed as a characteristic function $\mu_{A}(x)$ from $U$ in to $\{0,1\}$, such that:

$\mu_{A}(x)=\left\{\begin{array}{lll}1 & \text { if } & x \in A \\ 0 & \text { if } & x \notin A\end{array}\right.$

$\{0,1\}$ is called a valuation set. If the valuation set is allowed to be the real interval [0,1], then is called fuzzy set (which is denoted by $\tilde{A})$, and $\mu_{A}(x)$ is the grade of membership of $x$ in $\tilde{A}$.

Definition 2.2 [14]:

A Fuzzy number $(\widetilde{M})$ is a subset of the real line $\widetilde{M}: R \rightarrow[0,1]$, if it satisfies the following conditions:

(i) $\quad \widetilde{M}$ is normed, i.e. $\exists x_{0} \in R$ with $\mu_{\widetilde{M}}(x)=1$

(ii) $\quad \widetilde{M}$ is a Fuzzy convex, i.e.

$\mu_{\tilde{A}}(\lambda x+(1-\lambda) y) \geq \min \left\{\mu_{\tilde{A}}(x), \mu_{\tilde{A}}(y)\right\}, \forall \lambda \in[0,1], \forall x, y \in R$

(iii) $\widetilde{M}$ is upper semicontinuous on $R$ i.e. $\forall \varepsilon>0 \exists \delta>0$ such that $\left(\mu_{\widetilde{M}}(x)-\mu_{\widetilde{M}}\left(x_{0}\right)\right)<\varepsilon,\left|x-x_{0}\right|<\delta$

(iv) $\quad \widetilde{M}$ is compactly supported i.e. $\operatorname{cl}\left\{x \in R ; \mu_{\widetilde{M}}(x)>0\right\}$ is compact, where $c l(U)$ denoted the closure of the set $U$. This fuzzy number may be written as $M_{a}=[\underline{M}, \bar{M}]$, where $\underline{M}$ refers to the greatest lower bounded of $M_{a}$, and $\bar{M}$ to the least upper bounded of $M_{a}$ and Fuzzy center of an arbitrary Fuzzy number $\widetilde{M}=[\underline{M}, \bar{M}]$ is defined as $A^{c}=\left(\frac{\underline{M}+\bar{M}}{2}\right)$.

It is important to define and select a few special types of fuzzy numbers. Some such special types of fuzzy numbers are a triangular fuzzy number is represented by two end points $a_{1}$ and $a_{3}$ and a peak point $a_{2}$ as $\left[a_{1}, a_{2}, a_{3}\right]$, and a trapezoidal fuzzy number represented by two end points $a_{1}$ and $a_{4}$ and tolerance interval $\left[a_{2}, a_{3}\right]$ as $\left[a_{1}, a_{2}, a_{3}, a_{4}\right]$.

Definition 2.3 [15]:If $E$ be the set of all fuzzy number a mapping $y: T \rightarrow E$ for some interval $T \subseteq E$ is called fuzzy function process and denoted the $r-$ levelset by $[y(t)]_{r}=[\underline{y}(t ; r), \bar{y}(t ; r)] t \in T, r \in[0,1]$.

\section{DEFUZZIFICATION TECHNIQUES}

Extended Ranking Method based on philosophies of probability and ranking theories[16, 17]present a method for ordering fuzzy sets in which a ranking index $\mathfrak{R}(\widetilde{M})$ is calculated for the fuzzy number from its $r$-cut interval. For instant by considering the triangular fuzzy number $\widetilde{M}=\left(a_{1}, a_{2}, a_{3}\right)$, then $M_{r}=\left[a_{1}+\left(a_{2}-a_{1}\right) r, a_{3}-\left(a_{3}-a_{2}\right) r\right], 0 \leq r \leq 1$

$$
\begin{aligned}
\mathfrak{R}(\widetilde{M})= & \frac{1}{2}\left(\int_{0}^{1}\left(a_{1}+\left(a_{2}-a_{1}\right) r\right) d r+\int_{0}^{1}\left(a_{3}-\left(a_{3}-a_{2}\right) r\right) d r\right) \\
& \Re(\widetilde{M})=\frac{a_{1}+2 a_{2}+a_{3}}{4} .
\end{aligned}
$$

With the membership function

$\mu_{\widetilde{M}}(x)=\left\{\begin{array}{cc}\frac{x-a_{1}}{a_{2}-a_{1}}, & a_{1} \leq x \leq a_{2} \\ 1, & x=0 \\ \frac{a_{3}-x}{a_{3}-a_{2}}, & a_{2} \leq x \leq a_{3} \\ 0, & \text { otherwise }\end{array}\right.$

By setting $f(x)=c \mu_{\widetilde{M}}(x)$,

then 
$f(x)=\left\{\begin{array}{cc}\frac{2\left(x-a_{1}\right)}{\left(a_{3}-a_{1}\right)\left(a_{2}-a_{1}\right)}, & a_{1} \leq x<a_{2} \\ \frac{2}{a_{3}-a_{1}}, & x=a_{2} \\ \frac{2\left(a_{3}-x\right)}{\left(a_{3}-a_{1}\right)\left(a_{3}-a_{2}\right)}, & a_{2}<x \leq a_{3} \\ 0, & \text { otherwise. }\end{array}\right.$

$\int_{a_{1}}^{a_{3}} f(x) d x=\int_{a_{1}}^{a_{3}} c \mu_{\widetilde{M}}(x) d x=1$

then $c=\frac{2}{a_{3}-a_{1}}$ and $f_{1}(x), f_{2}(x)$ are linear functions

then we are obtained

$f_{1}(x)=\frac{2\left(x-a_{1}\right)}{\left(a_{3}+2 a_{2}-a_{1}\right)\left(a_{2}-a_{1}\right)}, \quad f_{2}(x)=\frac{2\left(a_{3}-x\right)}{\left(a_{3}+2 a_{2}-a_{1}\right)\left(a_{3}-a_{2}\right)}$

Then

$E_{1}(\widetilde{\bar{M}})=\int_{0}^{1} f_{1}^{-1}(\mu) d \mu=\frac{\left(a_{3}+2 a_{2}-a_{1}\right)\left(a_{2}-a_{1}\right)}{4}+a_{1}, \quad E_{2}(\widetilde{\bar{M}})=\int_{0}^{1} f_{2}^{-1}(\mu) d \mu=a_{3}-\frac{\left(a_{3}+2 a_{2}-a_{1}\right)\left(a_{3}-a_{2}\right)}{4}$

where $\widetilde{M}$ is denoted to the fuzzy stochastic variable of the fuzzy number $\widetilde{M}$

Then the expected interval of fuzzy stochastic variable $\widetilde{\bar{M}}$ can be expressed as: $E I(\widetilde{\bar{M}})=\left[E_{1}(\widetilde{\bar{M}}), E_{2}(\widetilde{\bar{M}})\right]$

If $f_{1}(x)$ and $f_{2}(x)$ are convex nonlinear.in this case, any approximated methods can be used to linearized $f_{1}(x), f_{2}(x)$, then the proposed the above method.

\section{VARIATIONAL ITERATION METHOD}

To illustrate the basic concept of Variational Iteration Method consider the following general equation:

$\frac{d^{m} y}{d t^{m}} R y(t)+N y(t)=g(t)$

whit initial conditions

$$
\left\{\begin{array}{l}
y(0)=(\underline{y}(0 ; r), \bar{y}(0 ; r)) \\
y^{\prime}(0)=\left(\underline{y}^{\prime}(0 ; r), \bar{y}^{\prime}(0 ; r)\right) \\
y^{\prime \prime}(0)=\left(\underline{y}^{\prime \prime}(0 ; r), \bar{y}^{\prime \prime}(0 ; r)\right) \\
\quad \vdots \\
y^{(m-1)}(0)=\left(\underline{y}^{(m-1)}(0 ; r), \bar{y}^{(m-1)}(0 ; r)\right)
\end{array}\right.
$$

Where $y(t)$ is fuzzy function, $g(t)$ is inhomogeneous source term, and $R, N$ are linear and nonlinear operators. The general Lagrange multiplier method was modified to an iteration method, which is known as correction functional. The basic character of the method is to construct a correction functional for equation (2), which reads as

$y_{n+1}(t)=y_{n}(t)+\int_{0}^{t} \lambda\left(\frac{d^{m} y}{d t^{m}} R y(t)+N y(t)-g(t)\right) d s$

Where, $\lambda$ is General Lagrange multiplier, which can be identified optimally via the variational theory, it is to be noted that $\lambda$ can be constant or a function.

And, the subscript $n$ denotes the nth approximation, and $\tilde{y}_{n}$ is the restricted variation i.e. $\delta \tilde{y}_{n}=0$.

To determine the Lagrange multiplier $\lambda$ that can be identified optimally via integration by parts and using a restricted variation $[12,13]$, that a general formula for $\lambda$ for the nth order differential equation is:

$y^{(m)}+f\left(y(t), y^{\prime}(t), y^{\prime \prime}(t), \ldots, y^{(m)}(t)\right)=0$ 
Lagrange multiplier can be proved as in the form bellows:

$\lambda=(-1)^{m} \frac{(s-t)^{m-1}}{(m-1) !}$

Take the determined of the Lagrange multiplier, the successive approximation $y_{n+1}$ is calculated by using any initial function $y_{0}$. Consequently, the solution obtained by taking the limit, as follows:

$y=\lim _{n \rightarrow \infty} y_{n}$

The correction function (5) is given a sequence of approximation, and the exact solution is obtained at the limit of the successive approximations. The solution of equation (3) is considered as a fixed point of the following functional under a suitable choice of the initial term of $y_{0}(t)$

\section{PROPOSED METHOD}

In this work, we are building a hybrid method based on the philosophy of combing Laplace transform method and variational iteration method presented in $[19,20]$ to develop our hybrid method. Since the variational iteration method depend on the value of the Lagrange multiplier, two different approaches for calculating the Lagrange multiplier are used to get more efficient method.

\section{First approach:}

1. After taking the Laplace transform of equation (2) yields:

$L\left(\frac{d^{m} y}{d t^{m}}\right)+L(R y(t)+N y(t))=L(g(t))$

By using the differential properties of Laplace transform and initial conditions that yields:

$$
\begin{aligned}
& \underline{y}_{n+1}(S)=\underline{y}_{n}(S)+\lambda(S)\left(S^{m} \underline{y}_{n}(S)-S^{m-1} \underline{y}(0)-\cdots-\underline{y}^{(m-1)}(0)+L\left[R\left(\underline{\tilde{y}}_{n}\right)+N\left(\underline{\tilde{y}}_{n}\right)-g(t)\right]\right) \\
& \bar{y}_{n+1}(S)=\bar{y}_{n}(S)+\lambda(S)\left(S^{m} \bar{y}_{n}(S)-S^{m-1} \bar{y}(0)-\cdots-\bar{y}^{(m-1)}(0)+L\left[R\left(\tilde{\bar{y}}_{n}\right)+N\left(\tilde{\bar{y}}_{n}\right)-g(t)\right]\right)
\end{aligned}
$$

2. The iteration formula of equations (8) and (9) can be used to suggest the main scheme involving the Lagrange multiplier. Consider the terms $L\left[R\left(\tilde{\bar{y}}_{n}\right)+N\left(\tilde{\bar{y}}_{n}\right)-g(t)\right]$ in equation (8) and $L\left[R\left(\tilde{\bar{y}}_{n}\right)+N\left(\tilde{\bar{y}}_{n}\right)-g(t)\right]$ in equation (9) as restricted variations, this makes equations (8) and (9) stationary with respect to $\underline{y}_{n}$ and $\bar{y}_{n}$ :

$\delta \underline{y}_{n+1}(S)=\delta \underline{y}_{n}(S)+\lambda(S)\left(S^{m} \delta \underline{y}_{n}(S)\right)$

$\delta \bar{y}_{n+1}(S)=\delta \bar{y}_{n}(S)+\lambda(S)\left(S^{m} \delta \bar{y}_{n}(S)\right)$

where $\delta$ is the classical variation, The optimality condition for the extreme $\frac{\delta y_{n+1}}{\delta y_{n}}=0$, Equations (10) and (11) lead to :

$$
\lambda(S)=\underline{\lambda}(S)=\bar{\lambda}(S)=-\frac{1}{S^{m}}
$$

3. The sequential approximations are obtained by taking the inverse Laplace transform to equations (8) and (9) after substituting $\lambda(S)$ yields:

$$
\begin{aligned}
& \underline{y}_{n+1}(t)=\underline{y}_{n}(t)-L^{-1}\left[\frac{1}{s^{m}}\left(S^{m} \underline{y}_{n}(s)-S^{m-1} \underline{y}(0)-\cdots-\underline{y}^{(m-1)}(0)+L\left[R\left(\underline{\tilde{y}}_{n}\right)+N\left(\underline{\tilde{y}}_{n}\right)-g(t)\right]\right)\right] \\
& \bar{y}_{n+1}(t)=\bar{y}_{n}(t)-L^{-1}\left[\frac{1}{s^{m}}\left(S^{m} \bar{y}_{n}(s)-S^{m-1} \bar{y}(0)-\cdots-\bar{y}^{(m-1)}(0)+L\left[R\left(\tilde{\bar{y}}_{n}\right)+N\left(\tilde{\bar{y}}_{n}\right)-g(t)\right]\right)\right]
\end{aligned}
$$

Rearrange equations (13) and (14) yields: 
$\underline{y}_{n+1}(t)=L^{-1}\left(\frac{\underline{y}(0)}{s}+\cdots+\frac{\underline{y}^{(m-1)}(0)}{s^{m}}\right)+L^{-1}\left[\frac{1}{s^{m}}\left(L\left[R\left(\underline{y}_{n}\right)+N\left(\underline{y}_{n}\right)-g(t)\right]\right)\right]$

$\bar{y}_{n+1}(t)=L^{-1}\left(\frac{\bar{y}(0)}{s}+\cdots+\frac{\bar{y}^{(m-1)}(0)}{s^{m}}\right)+L^{-1}\left[\frac{1}{s^{m}}\left(L\left[R\left(\bar{y}_{n}\right)+N\left(\bar{y}_{n}\right)-g(t)\right]\right)\right]$

With initial approximation to equations (15) and (16) the following equation can be considered:

$\underline{y}_{0}(t)=L^{-1}\left(\frac{\underline{y}(0)}{s}+\cdots+\frac{\underline{y}^{(m-1)}(0)}{s^{m}}\right)$

$\bar{y}_{0}(t)=L^{-1}\left(\frac{\bar{y}(0)}{s}+\cdots+\frac{\bar{y}^{(m-1)}(0)}{s^{m}}\right)$

after applying Laplace inverse to equations (15) and (16) yields:

$\underline{y}_{0}(t)=\underline{y}(0)+\underline{y}^{\prime}(0) t+\cdots+\frac{\underline{y}^{(m-1)}(0) t^{m-1}}{(m-1) !}$

$\bar{y}_{0}(t)=\bar{y}(0)+\bar{y}^{\prime}(0) t+\cdots+\frac{\bar{y}^{(m-1)}(0) t^{m-1}}{(m-1) !}$

4. Finally, the values of $y_{1}, y_{2}, y_{3}, \ldots, y_{n}$ are obtained, and the solution of equation (2) is:

$\underline{y}(\mathrm{t})=\lim _{n \rightarrow \infty} \underline{y}_{n}(t)$

$\bar{y}(\mathrm{t})=\lim _{n \rightarrow \infty} \bar{y}_{n}(t)$

\section{Second approach}

Consider the following general nonlinear equation

$\frac{d^{m} y}{d t^{m}}+R[y(t)]+N[y(t)]=g(t)$

$y^{(k)}(0)=\frac{d^{k} y(0)}{d t^{k}} \quad k=0,1, \ldots, m-1$

where $R$ is a linear operator, $N$ is nonlinear operator, $\mathrm{g}(\mathrm{t})$ is a nonhomogeneous

assuming that

$$
R[y(t)]=\sum_{i=0}^{m-1}\left(a_{i} y^{(i)}(t)+b_{i} y^{(i)}(t)\right)
$$

where $a_{i}$ constant and $b_{i}$ variable coefficient

1. Take the Laplace transform on (23), then the iteration formula becomes

$$
\begin{aligned}
& \underline{y}_{n+1}(s)=\underline{y_{n}}(s)+\lambda\left[s^{m} \underline{y}_{n}(s)-\sum_{k=0}^{m-1} \underline{y}^{(i)}(0) s^{m-k-1}+L\left(\sum_{i=0}^{m-1} a_{i} \underline{y}_{n}^{(i)}\right)+L\left(\sum_{i=0}^{m-1} b_{i}(t) \underline{y}_{n}^{(i)}\right)+L\left(N\left[\underline{y}_{n}\right]-g(t)\right)\right] \\
& \bar{y}_{n+1}(s)=\bar{y}_{n}(s)+\lambda\left[s^{m} \bar{y}_{n}(s)-\sum_{k=0}^{m-1} \bar{y}^{(i)}(0) s^{m-k-1}+L\left(\sum_{i=0}^{m-1} a_{i} \bar{y}_{n}^{(i)}\right)+L\left(\sum_{i=0}^{m-1} b_{i}(t) \bar{y}_{n}^{(i)}\right)+L\left(N\left[\bar{y}_{n}\right]-g(t)\right)\right]
\end{aligned}
$$

2. Consider the terms $L\left(\sum_{i=0}^{m-1} b_{i}(t) \underline{y}_{n}^{(i)}\right)$ and $L\left(N\left[\underline{y}_{n}\right]\right)$ in equation (25) and $L\left(\sum_{i=0}^{m-1} b_{i}(t) \bar{y}_{n}^{(i)}\right)$ and $L\left(N\left[\bar{y}_{n}\right]\right)$ in equation (26) as restricted variation. make equations (40) and (41) stationary with respect to $\underline{y}_{n}$ and $\bar{y}_{n}$ 
$\delta \underline{y}_{n+1}(s)=\delta \underline{y}_{n}(s)+\lambda\left[s^{m} \delta \underline{y}_{n}(s)+\sum_{i=0}^{m-1} a_{i} s^{i} \delta \underline{y}_{n}(S)\right]$

$\delta \bar{y}_{n+1}(s)=\delta \bar{y}_{n}(s)+\lambda\left[s^{m} \delta \bar{y}_{n}(s)+\sum_{i=0}^{m-1} a_{i} s^{i} \delta \bar{y}_{n}(S)\right]$

from equations (27) and (28) determined the Lagrange multiplier as follows

$\lambda(S)=\underline{\lambda}(S)=\bar{\lambda}(S)=-\frac{1}{\sum_{i=0}^{m-1} a_{i} s^{i}}, a_{m}=1$

3. Take the Laplace inverse for equations (25) and (26) then the iteration formula obtained as

$\underline{y}_{n+1}(t)=\underline{y}_{n}(s)+L^{-1}\left[\lambda(S)\left[s^{m} \underline{y}_{n}(s)-\sum_{k=0}^{m-1} \underline{y}^{(i)}(0) s^{m-k-1}+L\left(\sum_{i=0}^{m-1} a_{i} \underline{y}_{n}^{(i)}\right)+L\left(\sum_{i=0}^{m-1} b_{i}(t) \underline{y}_{n}^{(i)}\right)+L\left(N\left[\underline{y}_{n}\right]-\right.\right.\right.$ $g(t))]]$

$\bar{y}_{n+1}(t)=\bar{y}_{n}(s)+L^{-1}\left[\lambda(S)\left[s^{m} \bar{y}_{n}(s)-\sum_{k=0}^{m-1} \bar{y}^{(i)}(0) s^{m-k-1}+L\left(\sum_{i=0}^{m-1} a_{i} \bar{y}_{n}^{(i)}\right)+L\left(\sum_{i=0}^{m-1} b_{i}(t) \bar{y}_{n}^{(i)}\right)+L\left(N\left[\bar{y}_{n}\right]-\right.\right.\right.$ $g(t))]]$

$\underline{y}_{n+1}(t)=\underline{y}_{0}+L^{-1}\left(\sum_{i=0}^{m-1} b_{i}(t) \underline{y}_{n}^{(i)}\right)+N\left[\underline{y}_{n}\right]$

$\bar{y}_{n+1}(t)=\bar{y}_{0}+L^{-1}\left(\sum_{i=0}^{m-1} b_{i}(t) \bar{y}_{n}^{(i)}\right)+N\left[\bar{y}_{n}\right]$

where the initial iteration value can be determined as

$\underline{y}_{0}(t)=L^{-1}\left[\lambda(s)\left[-\sum_{k=0}^{m-1} \underline{y}^{(i)}(0) s^{m-k-1}+L\left(\sum_{i=0}^{m-1} a_{i} \underline{y}_{n}^{(i)}\right)++L[g(t)]\right]\right]$

$\bar{y}_{0}(t)=L^{-1}\left[\lambda(s)\left[-\sum_{k=0}^{m-1} \bar{y}^{(i)}(0) s^{m-k-1}+L\left(\sum_{i=0}^{m-1} a_{i} \bar{y}_{n}^{(i)}\right)++L[g(t)]\right]\right]$

4. Let $y_{n}=\sum_{j=0}^{n} v_{j}$ and apply the Adomian decomposition method (ADM) [8] to expand the term $N\left[y_{n}\right]$ as $\sum_{j=0}^{n} A_{j}$ then the iteration formula

$\left\{\begin{array}{l}v_{j+1}=L^{-1}\left(\sum_{i=0}^{m-1} b_{i}(t) v_{j}^{(i)}\right)+A_{j} \\ v_{0}=y_{0}\end{array}\right.$

where $A_{j}$ is the famous Adomian decomposition series.

Example (3.1): The example solved in [21] can be resolved by the hybrid VIM that proposed by this work.

Consider the following second-order linear fuzzy differential equation.

$\left\{\begin{array}{l}y^{\prime \prime}(t)+y(t)=-t \quad t \in[0,1] \\ y(0)=(0.1 r-0.1,0.1-0.1 r) \\ y^{\prime}(0)=(0.088+0.1 r, 0.288-0.1 r)\end{array}\right.$

The solution of equation (37) is using hybrid method is as follows:

\section{First approach:}

Take the Laplace transform to the equation yields:

$\mathrm{s}^{2} \mathrm{y}(\mathrm{s})-\mathrm{sy}(0)-\mathrm{y}^{\prime}(0)=\mathrm{L}[-\mathrm{y}-\mathrm{t}]$

Obtain the iteration formula of equation (38) yields:

$\mathrm{y}_{\mathrm{n}+1}(\mathrm{~s})=\mathrm{y}_{\mathrm{n}}(\mathrm{s})+\lambda(\mathrm{s})\left[\mathrm{s}^{2} \mathrm{y}_{\mathrm{n}}(\mathrm{s})-\mathrm{sy}(0)-\mathrm{y}^{\prime}(0)+\mathrm{y}_{\mathrm{n}}(\mathrm{s})+\frac{1}{\mathrm{~s}}\right]$

Consider the term $\left(y_{n}(s)+\frac{1}{s}\right)$ in the above equation as restricted variation, then:

$\delta y_{n+1}=\delta y_{n}(s)+\lambda(s)\left(s^{2} \delta y_{n}(s)\right)$

With the Lagrange multiplier of equation (39) is: 
$\lambda(s)=-\frac{1}{s^{2}}$

Taking the Laplace inverse transform to equation (39):

$\mathrm{y}_{\mathrm{n}+1}(\mathrm{t})=\mathrm{y}_{\mathrm{n}}(\mathrm{t})-\mathrm{L}^{-1}\left\{\frac{1}{\mathrm{~s}^{2}}\left[\mathrm{~s}^{2} \mathrm{y}_{\mathrm{n}}(\mathrm{s})-\mathrm{sy}(0)-\mathrm{y}^{\prime}(0)+\mathrm{y}_{\mathrm{n}}(\mathrm{s})+\frac{1}{\mathrm{~s}}\right]\right\}$

$y_{n+1}(t)=L^{-1}\left[\frac{y(0)}{s}+\frac{y^{\prime}(0)}{s^{2}}\right]+L^{-1}\left\{\frac{1}{s^{2}}\left[y_{n}(s)+\frac{1}{s}\right]\right\}$

Therefore,

$\mathrm{y}_{\mathrm{n}+1}(\mathrm{t})=\mathrm{y}_{0}(\mathrm{t})+\mathrm{L}^{-1}\left\{\frac{1}{\mathrm{~s}^{2}}\left[\mathrm{y}_{\mathrm{n}}(\mathrm{s})+\frac{1}{\mathrm{~s}}\right]\right\}$

With initial iteration

$\mathrm{y}_{0}(\mathrm{t})=\mathrm{y}(0)+\mathrm{y}^{\prime}(0) \mathrm{t}$

Case (1): according to $y(t)$ the following solution can be obtained:

With exact fuzzy $y(t, r)$

$\underline{y}(t, r)=(0.1 r-0.1) \cos t+(1.088+0.1 r) \sin t-t$

The initial conditions:

$\underline{\mathrm{y}}(0)=(0.1 \mathrm{r}-0.1)$

Using the Defuzzification techniques $\mathrm{E}_{1}=\int_{0}^{1}(0.1 \mathrm{r}-0.1) \mathrm{dr}=-0.05$ yields:

$\mathrm{y}(0)=-0.05$

$\overline{\mathrm{y}}^{\prime}(0)=(0.088+0.1 \mathrm{r})$

Using the Defuzzification techniques $\mathrm{E}_{1}=\int_{0}^{1}(0.088+0.1 \mathrm{r}) \mathrm{dr}=0.138 \mathrm{yields}$ :

$\mathrm{y}^{\prime}(0)=0.138$

then

$\underline{\mathrm{y}}_{0}(\mathrm{t})=-0.05+0.138 \mathrm{t}$

$\underline{\mathrm{y}}_{1}(\mathrm{t})=-0.05+0.138 \mathrm{t}+\frac{0.05}{2 !} \mathrm{t}^{2}-\frac{0.138}{3 !} \mathrm{t}^{3}-\frac{\mathrm{t}^{3}}{3 !}$

$\underline{\mathrm{y}}_{2}(\mathrm{t})=-0.05+0.138 \mathrm{t}+\frac{0.05}{2 !} \mathrm{t}^{2}-\frac{0.138}{3 !} \mathrm{t}^{3}-\frac{0.05}{4 !} \mathrm{t}^{4}+\frac{0.138}{5 !} \mathrm{t}^{5}-\frac{\mathrm{t}^{3}}{3 !}+\frac{\mathrm{t}^{5}}{5 !}$

$\underline{y}_{3}(t)=-0.05+0.138 t+\frac{0.05}{2 !} t^{2}-\frac{0.138}{3 !} t^{3}-\frac{0.05}{4 !} t^{4}+\frac{0.138}{5 !} t^{5}+\frac{0.05}{6 !} t^{6}-\frac{0.138}{7 !} t^{7}-\frac{t^{3}}{3 !}+\frac{t^{5}}{5 !}-\frac{t^{7}}{7 !}$

!

$\underline{\mathrm{y}}_{\mathrm{n}}(\mathrm{t})=-0.05+\frac{0.05}{2 !} \mathrm{t}^{2}-\frac{0.05}{4 !} \mathrm{t}^{4}+\frac{0.05}{6 !} \mathrm{t}^{6}-\cdots \pm \frac{0.05}{2 \mathrm{n} !} \mathrm{t}^{2 \mathrm{n}}+0.138 \mathrm{t}-\frac{0.138}{3 !} \mathrm{t}^{3} \frac{0.138}{5 !} \mathrm{t}^{5}-\frac{0.138}{7 !} \mathrm{t}^{7}+\cdots \pm \frac{0.138}{2 \mathrm{n}+1 !} \mathrm{t}^{2 \mathrm{n}+1}-\frac{\mathrm{t}^{3}}{3 !}+\frac{\mathrm{t}^{5}}{5 !}-$ $\frac{\mathrm{t}^{7}}{7 !}+\cdots \pm \frac{\mathrm{t}^{2 \mathrm{n}+1}}{2 \mathrm{n}+1 !}$

As $\mathrm{n} \rightarrow \infty \mathrm{y}_{\mathrm{n}}(\mathrm{t}), \underline{\mathrm{y}}(\mathrm{t})$ converge to $-0.05 \cos \mathrm{t}+1.138 \sin \mathrm{t}-\mathrm{t}$

The exact solution is shown in Figure 1.

Case (2): according to $\bar{y}$ the following solution can be obtained

With exact fuzzy $\bar{y}(t, r)$

$\bar{y}(t, r)=(0.1-0.1 r) \cos t+(1.288+0.1 r) \sin t-t$

The initial conditions:

$\overline{\mathrm{y}}(0)=(0.1-0.1 \mathrm{r})$

Using the Defuzzification techniques $\mathrm{E}_{2}=\int_{0}^{1}(0.1-0.1 \mathrm{r}) \mathrm{dr}=0.05$ yields:

$\overline{\mathrm{y}}(0)=0.05$

$\overline{\mathrm{y}}^{\prime}(0)=(0.288-0.1 \mathrm{r})$

Using the Defuzzification techniques $\mathrm{E}_{2}=\int_{0}^{1}(0.288-0.1 \mathrm{r}) \mathrm{dr}=0.238$ yields:

$\overline{\mathrm{y}}^{\prime}(0)=0.238$

then

$\overline{\mathrm{y}}_{0}(\mathrm{t})=0.05+0.238 \mathrm{t}$

$\bar{y}_{1}(t)==0.05+0.238 t-\frac{0.05}{2 !} t^{2}-\frac{0.238}{3 !} t^{3}-\frac{t^{3}}{3 !}$

$\bar{y}_{2}(t)=0.05+0.238 t-\frac{0.05}{2 !} t^{2}-\frac{0.238}{3 !} t^{3}+\frac{0.05}{4 !} t^{4}+\frac{0.238}{5 !} t^{5}-\frac{t^{3}}{3 !}+\frac{t^{5}}{5 !}$

$\bar{y}_{3}(t)=0.05+0.238 t-\frac{0.05}{2 !} t^{2}-\frac{0.238}{3 !} t^{3}+\frac{0.05}{4 !} t^{4}+\frac{0.238}{5 !} t^{5}-\frac{0.05}{6 !} t^{6}-\frac{0.238}{7 !} t^{7}-\frac{t^{3}}{3 !}+\frac{t^{5}}{5 !}-\frac{t^{7}}{7 !}$ 
$\bar{y}_{n}(t)=0.05-\frac{0.05}{2 !} t^{2}+\frac{0.05}{4 !} t^{4}-\frac{0.05}{6 !} t^{6}+\cdots \pm \frac{0.05}{2 n !} t^{2 n}+0.238 t-\frac{0.238}{3 !} t^{3}+\frac{0.238}{5 !} t^{5}-\frac{0.238}{7 !} t^{7}+\cdots \pm \frac{0.238}{2 n+1 !} t^{2 n+1}-\frac{t^{3}}{3 !}+\frac{t^{5}}{5 !}-$ $\frac{\mathrm{t}^{7}}{7 !}+\cdots \pm \frac{\mathrm{t}^{2 \mathrm{n}+1}}{2 \mathrm{n}+1 !}$

As $\rightarrow \infty \bar{y}_{n}(t), \bar{y}(t)$ converge to $0.05 \cos t+1.238 \sin t-t$

Figure 2 shows the exact solution at $r=1$ and the approximate solution.

Case (3): according to $\mathrm{y}^{\mathrm{c}}$ the following solution can be obtained:

with exact fuzzy $\mathrm{y}^{\mathrm{c}}(\mathrm{t}, \mathrm{r})$

$\mathrm{y}^{\mathrm{c}}(\mathrm{t}, \mathrm{r})=1.188 \sin \mathrm{t}-\mathrm{t}$

Using the initial condition:

$\mathrm{y}^{\mathrm{c}}(0)=\frac{(0.1 \mathrm{r}-0.1+0.1-0.1 \mathrm{r})}{2}=0$

$\mathrm{y}^{\mathrm{c}^{\prime}}(0)=\frac{(0.088+0.1 \mathrm{r}+0.288-0.1 \mathrm{r})}{2}=0.188$

then

$\mathrm{y}_{0}{ }^{\mathrm{c}}(\mathrm{t})=0.188 \mathrm{t}$

$\mathrm{y}_{1}^{\mathrm{c}}(\mathrm{t})=0.188 \mathrm{t}-\frac{0.188}{3 !} \mathrm{t}^{3}-\frac{\mathrm{t}^{3}}{3 !}$

$y_{2}^{c}(t)=0.188 t-\frac{0.188}{3 !} t^{3}+\frac{0.188}{5 !} t^{5}-\frac{t^{3}}{3 !}+\frac{t^{5}}{5 !}$

$\mathrm{y}_{3}^{\mathrm{c}}(\mathrm{t})=0.188 \mathrm{t}-\frac{0.188}{3 !} \mathrm{t}^{3}+\frac{0.188}{5 !} \mathrm{t}^{5}-\frac{0.188}{7 !} \mathrm{t}^{7}-\frac{\mathrm{t}^{3}}{3 !}+\frac{\mathrm{t}^{5}}{5 !}-\frac{\mathrm{t}^{7}}{7 !}$

$y_{n}^{c}(t)=0.188 \mathrm{t}-\frac{0.188}{3 !} \mathrm{t}^{3}+\frac{0.188}{5 !} \mathrm{t}^{5}-\frac{0.188}{7 !} \mathrm{t}^{7}+\cdots \pm \frac{0.188}{2 \mathrm{n}+1 !} \mathrm{t}^{2 \mathrm{n}+1}-\frac{\mathrm{t}^{3}}{3 !}+\frac{\mathrm{t}^{5}}{5 !}-\frac{\mathrm{t}^{7}}{7 !}+\cdots \pm \frac{\mathrm{t}^{2 \mathrm{n}+1}}{2 \mathrm{n}+1 !}$

As $\rightarrow \infty \mathrm{y}_{\mathrm{n}}^{\mathrm{c}}(\mathrm{t}), \mathrm{y}^{\mathrm{c}}(\mathrm{t})$ converge to $1.188 \sin \mathrm{t}-\mathrm{t}$

The exact solution is shown in Figure 3.

Second approach:

Take the Laplace transform to the equation (37) yields:

$\mathrm{s}^{2} \mathrm{y}(\mathrm{S})-\mathrm{sy}(0)-\mathrm{y}^{\prime}(0)+\mathrm{L}[\mathrm{y}+\mathrm{t}]=0$

Obtain the iteration formula of equation (3.87) yields:

$\mathrm{y}_{\mathrm{n}+1}(\mathrm{~s})=\mathrm{y}_{\mathrm{n}}(\mathrm{s})+\lambda\left[\mathrm{s}^{2} \mathrm{y}(\mathrm{s})-\mathrm{sy}(0)-\mathrm{y}^{\prime}(0)+\mathrm{y}(\mathrm{s})+\frac{1}{\mathrm{~s}^{2}}\right]$

Consider the term $\frac{1}{\mathrm{~s}^{2}}$ as restricted variation

$\delta y_{n+1}=\delta y_{n}(s)+\lambda(s)\left(s^{2}-1\right) \delta y_{n}(s)$

with the Lagrange multiplier

$\lambda(\mathrm{s})=-\frac{1}{\sum_{\mathrm{i}=0}^{2} \mathrm{a}_{\mathrm{i}} \mathrm{s}^{\mathrm{i}}}=-\frac{1}{\mathrm{~s}^{2}+1}$

substitute $\lambda(\mathrm{s})$ in equation (53) and Take the Laplace inverse, yields:

$y_{n+1}(t)=L^{-1}\left[\frac{1}{s^{2}+1}\left[\operatorname{sy}(0)+y^{\prime}(0)\right]\right]+L^{-1}\left[\frac{-1}{\left(s^{2}+1\right)}\left(\frac{1}{s^{2}}\right)\right]$

$y_{n+1}(t)=y_{0}+L^{-1}\left[\frac{-1}{s^{2}}+\frac{1}{\left(s^{2}+1\right)}\right]$

where

$\mathrm{y}_{0}=\mathrm{L}^{-1}\left[\frac{1}{\mathrm{~S}^{2}+1}\left[\mathrm{Sy}(0)+\mathrm{y}^{\prime}(0)\right]\right]$

Case (1): according to $\underline{y}(\mathrm{t})$ the following solution can be obtained:

using the initial condition as equations (43) and (44)

$\underline{y}(0)=-0.05, \underline{y}^{\prime}(0)=0.138$

then

$\underline{\mathrm{y}}_{0}=\mathrm{L}^{-1}\left[\frac{\mathrm{s}(-0.05)}{\mathrm{s}^{2}+1}+\frac{0.138}{\mathrm{~s}^{2}+1}\right]=-0.5 \cos (\mathrm{t})+0.138 \sin (\mathrm{t})$

$\mathrm{y}_{1}(\mathrm{t})=-0.05 \cos (\mathrm{t})+1.138 \sin (\mathrm{t})-\mathrm{t}$

Case (2): according to $\bar{y}$ the following solution can be obtained using the initial condition yields as in equation (46) and (47):

$\overline{\mathrm{y}}(0)=0.05, \overline{\mathrm{y}}^{\prime}(0)=0.238$

then

$\overline{\mathrm{y}}_{0}=\mathrm{L}^{-1}\left[\frac{\mathrm{s}(0.05)}{\mathrm{s}^{2}+1}+\frac{0.238}{\mathrm{~s}^{2}+1}\right]=0.5 \cos (\mathrm{t})+0.238 \sin (\mathrm{t})$

$\bar{y}_{1}(t)=0.5 \cos (\mathrm{t})+1.238 \sin (\mathrm{t})-\mathrm{t}$ 
Case (3): according to $y^{c}$ the following solution can be obtained using the initial condition as in equation (49) and (59) $\mathrm{y}^{\mathrm{c}}(0)=0, \mathrm{y}^{\mathrm{c}^{\prime}}(0)=0.188$

then

$\mathrm{y}_{0}^{\mathrm{c}}=\mathrm{L}^{-1}\left[\frac{0.188}{\mathrm{~s}^{2}+1}\right]=0.188 \sin (\mathrm{t})$

$\mathrm{y}_{1}^{\mathrm{c}}(\mathrm{t})=1.88 \sin (\mathrm{t})-\mathrm{t}$

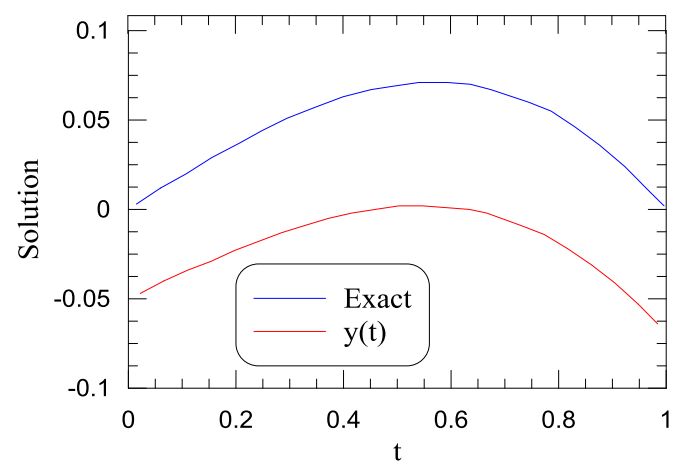

Figure 1: The exact solution of $y$ at $r=1$ and the approximate solution of $\mathrm{y}(\mathrm{t})$ by hybrid method

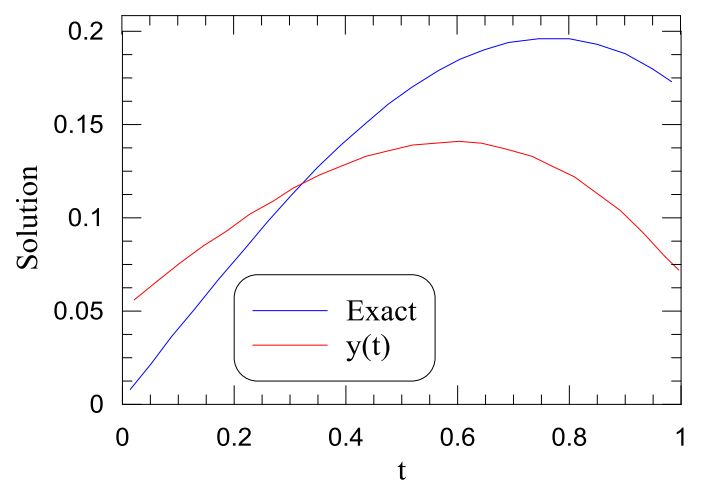

Figure 2: The exact solution of $\bar{y}$ at $r=1$ and the approximate solution of $\bar{y}(t)$ by hybrid method

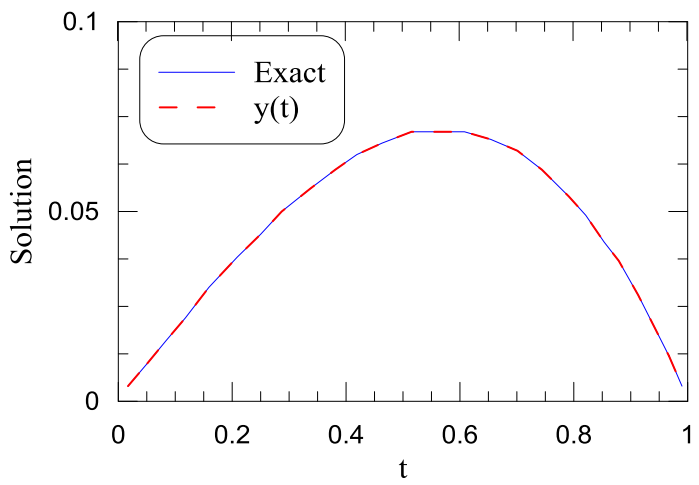

Figure 3: The exact solution of $y^{c}$ and the approximate solution of $y^{c}(t)$ by hybrid method

Error $=[(E)]_{r}=[\underline{E}, \bar{E}]=\left|\int_{0}^{1} \operatorname{exact}(t ; r)-y(t)\right| d t$

Table1: Show absolute error for the lower and upper approximate

\begin{tabular}{ccc}
\hline$r$ & Error for the lower $(\underline{E})$ & Error for the upper $(\bar{E})$ \\
\hline 0 & $8.702 \times 10^{-4}$ & $8.8215 \times 10^{-4}$ \\
0.1 & $6.9555 \times 10^{-4}$ & $7.0917 \times 10^{-4}$ \\
0.2 & $5.209 \times 10^{-4}$ & $5.3619 \times 10^{-4}$ \\
0.3 & $3.4625 \times 10^{-4}$ & $3.6321 \times 10^{-4}$ \\
0.4 & $1.716 \times 10^{-4}$ & $1.9023 \times 10^{-4}$ \\
0.5 & $3.05 \times 10^{-6}$ & $4.497 \times 10^{-4}$ \\
0.6 & $1.777 \times 10^{-4}$ & $1.5573 \times 10^{-4}$ \\
0.7 & $3.5235 \times 10^{-4}$ & $3.2871 \times 10^{-4}$ \\
0.8 & $5.27 \times 10^{-4}$ & $5.0169 \times 10^{-4}$ \\
0.9 & $7.0165 \times 10^{-4}$ & $6.7467 \times 10^{-4}$ \\
1 & $8.763 \times 10^{-4}$ & $8.4765 \times 10^{-4}$ \\
\hline
\end{tabular}

\section{CONCLUSION}

In this paper Laplace transformation and variational iteration method are used to obtain the approximation solutions of linear and nonlinear fuzzy differential equations. In this work, two approaches are building according to the type of the 
identification of the Lagrange multipliers. The two approaches obtained the same results in which the second approach consume more simple mathematical operations. Comparing with the exact solutions, the comparison table had been shown the difference is nearly to zero $\left(1.716 \times 10^{-4}\right)$ in lower and upper solutions while the result exactly the same in center solution. In general our results had been shown more accurate than the results obtained by others and consume less number of iteration compared with the other method.

\section{REFERENCES}

[1] L. A. Zadeh, "Fuzzy sets," Inf. Control, vol. 8, no. 3, pp. 338-353, Jun. 1965.

[2] O. Kaleva, "Fuzzy differential equations," Fuzzy Sets Syst., vol. 24, no. 3, pp. 301-317, Dec. 1987.

[3] A. Khastan and J. J. Nieto, "A boundary value problem for second order fuzzy differential equations," Nonlinear Anal. Theory Methods Appl., vol. 72, no. 9-10, pp. 3583-3593, May 2010.

[4] L. Ahmad, M. Farooq, S. Ullah, and S. Abdullah, "Solving fuzzy two-point boundary value problem using fuzzy Laplace transform," ArXiv Prepr. ArXiv14030571, 2014.

[5] L. Ahmad, M. Farooq, and S. Abdullah, "Solving nth order fuzzy differential equation by fuzzy Laplace transform," ArXiv14030242 Math, Mar. 2014.

[6] M. Ghanbari, "Numerical solution of fuzzy initial value problems under generalized di erentiability by HPM," Int. J. Ind. Math., vol. 1, no. 1, pp. 19-39, 2009.

[7] E. Babolian, H. Sadeghi, and S. Javadi, "Numerically solution of fuzzy differential equations by Adomian method," Appl. Math. Comput., vol. 149, no. 2, pp. 547-557, Feb. 2004.

[8] G. Adomian, "A review of the decomposition method in applied mathematics," J. Math. Anal. Appl., vol. 135, no. 2, pp. 501-544, Nov. 1988.

[9] T. Allahviranloo, S. Khezerloo, and M. Mohammadzaki, "Numerical solution for differential inclusion by adomian decomposition method," J. Appl. Math., vol. 5, no. 17, pp. 51-62, 2008.

[10] J.-H. He, "Variational iteration method - a kind of non-linear analytical technique: some examples," Int. J. Non-Linear Mech., vol. 34, no. 4, pp. 699-708, Jul. 1999.

[11] J.-H. He, "Some asymptotic methods for strongly nonlinear equations," Int. J. Mod. Phys. B, vol. 20, no. 10, pp. 11411199, Apr. 2006.

[12] H. Jafari, M. Saeidy, and D. Baleanu, "The variational iteration method for solving $\mathrm{n}$-th order fuzzy differential equations," Cent. Eur. J. Phys., vol. 10, no. 1, pp. 76-85, Feb. 2012.

[13] D. J. Dubois, Fuzzy Sets and Systems: Theory and Applications. Academic Press, 1980.

[14] P. Diamond and P. Kloeden, "Metric Topology of Fuzzy Numbers and Fuzzy Analysis," in Fundamentals of Fuzzy Sets, D. Dubois and H. Prade, Eds. Springer US, 2000, pp. 583-641.

[15] O. S. Fard, "An iterative scheme for the solution of generalized system of linear fuzzy differential equations," World Appl. Sci. J., vol. 7, no. 12, pp. 1597-1604, 2009.

[16] N. A. Alaulden and M. Y. Sanar, "Solving Fuzzy Network Problems by Defuzzification Techniques," Int. J. Innov. Res. Sci. Eng. Technol., 2014.

[17] R. R. Yager, "A procedure for ordering fuzzy subsets of the unit interval,” Inf. Sci., vol. 24, no. 2, pp. 143-161, Jul. 1981.

[18] H. Jafari and A. Alipoor, "A new method for calculating general lagrange multiplier in the variational iteration method," Numer. Methods Partial Differ. Equ., vol. 27, no. 4, pp. 996-1001, Jul. 2011.

[19] G.-C. Wu and D. Baleanu, "Variational iteration method for fractional calculus - a universal approach by Laplace transform," Adv. Differ. Equ., vol. 2013, no. 1, p. 18, Dec. 2013.

[20] G.-C. Wu, "Challenge in the variational iteration method - A new approach to identification of the Lagrange multipliers," J. King Saud Univ. - Sci., vol. 25, no. 2, pp. 175-178, Apr. 2013.

[21] H. Jafari, M. Saeidy, and D. Baleanu, "The variational iteration method for solving n-th order fuzzy differential equations," Open Phys., vol. 10, no. 1, pp. 76-85, 2011. 